\title{
Figur- und gewichtsbezogene Sorgen bei Frauen im mittleren Lebensalter und der Zusammenhang mit negativer Affektivität und Bildung
}

\author{
Drobnjak, Suzana ; Ehlert, Ulrike
}

\begin{abstract}
Hintergrund: Der Zusammenhang zwischen negativer Affektivität, Bildung und den figur- und gewichtsbezogenen Sorgen ist für junge Frauen weitgehend erforscht. Hingegen gibt es kaum Studien, welche die entsprechenden Faktoren bei Frauen im mittleren Lebensalter untersuchen. Ziel dieser Studie war es zu prüfen, ob es einen Zusammenhang zwischen Depressivität, Ängstlichkeit, zwanghaftem Verhalten und den figur- und gewichtsbezogenen Sorgen bei Frauen im mittleren Lebensalter gibt. Zudem wurde die Rolle des Bildungsniveaus untersucht. Methoden: Im Rahmen einer Online-Untersuchung zum Essverhalten bei Frauen über 40 Jahren wurden, neben sozioökonomischen Variablen, die figur- und gewichtsbezogenen Sorgen der Teilnehmerinnen mittels einer validierten deutschen Version des «Eating Disorder Examination Questionnaire» (EDE-Q) erfasst. Zusätzlich wurde eine deutsche Version des «Brief Symptom Inventory»(BSI) eingesetzt. In die Datenanalyse wurden die Ergebnisse von 550 Frauen im Alter von 40 bis 65 Jahren einbezogen. Ergebnisse: Depressivität, Ängstlichkeit und Zwanghaftigkeit korrelieren positiv mit figur- und gewichtsbezogenen Sorgen. Bei Frauen mit einem niedrigeren Bildungsniveau war der Zusammenhang zwischen Depressivität und figur- und gewichtsbezogenen Sorgen höher als bei Frauen mit einem höheren Bildungsniveau. Berechnungen anhand linearer Regressionsanalysen zeigten, dass Depressivität und Zwanghaftigkeit die stärksten Prädiktoren für figur- und essensbezogene Sorgen für beide Bildungsgruppen waren. Diskussion: Wenngleich die Ergebnisse dieser Studie bei gesunden Frauen im mittleren Lebensalter erhoben wurden, gibt der beobachtete Zusammenhang zwischen negativer Affektivität, figur- und gewichtsbezogenen Sorgen und Bildung Hinweise auf eine Frühindikation bzw. Prävention von Essstörungen.
\end{abstract}

DOI: https://doi.org/10.1159/000342173

Posted at the Zurich Open Repository and Archive, University of Zurich

ZORA URL: https://doi.org/10.5167/uzh-65291

Journal Article

Published Version

Originally published at:

Drobnjak, Suzana; Ehlert, Ulrike (2012). Figur- und gewichtsbezogene Sorgen bei Frauen im mittleren Lebensalter und der Zusammenhang mit negativer Affektivität und Bildung. Verhaltenstherapie, $22(3): 165-171$

DOI: https://doi.org/10.1159/000342173 


\title{
Figur- und gewichtsbezogene Sorgen bei Frauen im mittleren Lebensalter und der Zusammenhang mit negativer Affektivität und Bildung
}

\author{
Suzana Drobnjak Ulrike Ehlert \\ Abteilung Klinische Psychologie und Psychotherapie, Psychologisches Institut, \\ Universität Zürich, Schweiz
}

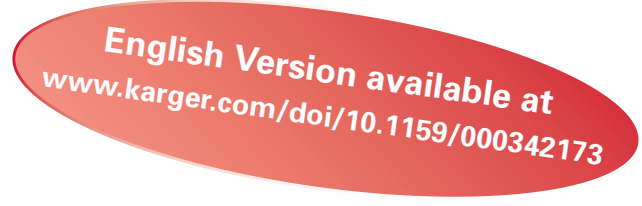

\section{Schlüsselwörter}

Figurbezogene Sorgen - Gewichtsbezogene Sorgen • Depressivität · Ängstlichkeit · Zwanghafte Züge · Bildung

\section{Zusammenfassung}

Hintergrund: Der Zusammenhang zwischen negativer Affektivität, Bildung und den figur- und gewichtsbezogenen Sorgen ist für junge Frauen weitgehend erforscht. Hingegen gibt es kaum Studien, welche die entsprechenden Faktoren bei Frauen im mittleren Lebensalter untersuchen. Ziel dieser Studie war es zu prüfen, ob es einen Zusammenhang zwischen Depressivität, Ängstlichkeit, zwanghaftem Verhalten und den figur- und gewichtsbezogenen Sorgen bei Frauen im mittleren Lebensalter gibt. Zudem wurde die Rolle des Bildungsniveaus untersucht. Methoden: Im Rahmen einer Online-Untersuchung zum Essverhalten bei Frauen über 40 Jahren wurden, neben sozioökonomischen Variablen, die figur- und gewichtsbezogenen Sorgen der Teilnehmerinnen mittels einer validierten deutschen Version des «Eating Disorder Examination Questionnaire" (EDE-Q) erfasst. Zusätzlich wurde eine deutsche Version des "Brief Symptom Inventory" (BSI) eingesetzt. In die Datenanalyse wurden die Ergebnisse von 550 Frauen im Alter von 40 bis 65 Jahren einbezogen. Ergebnisse: Depressivität, Ängstlichkeit und Zwanghaftigkeit korrelieren positiv mit figur- und gewichtsbezogenen Sorgen. Bei Frauen mit einem niedrigeren Bildungsniveau war der Zusammenhang zwischen Depressivität und figur- und gewichtsbezogenen Sorgen höher als bei Frauen mit einem höheren Bildungsniveau. Berechnungen anhand linearer Regressionsanalysen zeigten, dass Depressivität und Zwanghaftigkeit die stärksten Prädiktoren für figur- und essensbezogene Sorgen für beide Bildungsgruppen waren. Diskussion: Wenngleich die Ergebnisse dieser Studie bei gesunden Frauen im mittleren Lebensalter erhoben wurden, gibt der beobachtete $\mathrm{Zu}$ sammenhang zwischen negativer Affektivität, figur- und gewichtsbezogenen Sorgen und Bildung Hinweise auf eine Frühindikation bzw. Prävention von Essstörungen.

\section{Keywords}

Shape concern - Weight concern - Depressive mood . Anxiety · Obsessiveness · Education

\section{Summary}

Shape and Weight Concerns in Middle-Aged Women and the Relationship to Negative Affect and Education

Background: The relationship between negative affect, education, and shape and weight concerns has been well researched for young women. However, there have been no studies to investigate the respective factors in middle-aged women. The goal of this study was to examine whether there is a relationship between depression, anxiety, obsessiveness and shape and weight concerns in middle-aged women and to what extent the level of education may play a role. Methods: We used an online version of the 'Eating Disorder Examination Questionnaire' (EDE-O) to assess subjective ratings on eating behavior. Furthermore, the women rated their mood, level of anxiety and obsessiveness on a German version of the 'Brief Symptom Inventory' (BSI). We included 550 women in our data analysis between 40 and 65 years of age. Results: Depression, anxiety and obsessiveness correlate positively with shape and weight concerns. The relationship between depression and shape and weight concerns was stronger in women with a lower educational level. According to linear regression models, depression and obsessiveness were best predictors for shape and weight concerns for both educational levels. Discussion: The study examined healthy women in middle age. The relationship between negative affect, shape and weight concerns and educational level could nevertheless be used in the prevention or early indication of eating disorders.

\section{KARGER \\ Fax +497614520714 \\ Information@Karger.de}

www.karger.com (c) 2012 S. Karger GmbH, Freiburg

$1016-6262 / 12 / 0223-0165 \$ 38.00 / 0$

Accessible online at:

www.karger.com/ver
Prof. Dr. Ulrike Ehlert

Abteilung Klinische Psychologie und Psychotherapie, Psychologisches Institut Universität Zürich

Binzmühlestrasse 14 / Box 26, 8050 Zürich, Schweiz

Tel. $+414463573-50$

u.ehlert@psychologie.uzh.ch 


\section{Hintergrund}

Essstörungen sind ein Thema, das in der Öffentlichkeit und in der Wissenschaft seit Jahren große Aufmerksamkeit erfährt. Die Bemühungen, diese Störungen zu verstehen, haben sich bisher mehrheitlich auf junge Frauen bezogen [APA, 2000]. Das Essverhalten von Frauen im mittleren Lebensalter, also der Zeitspanne zwischen dem 40. und 65. Lebensjahr [Augustus-Horvath und Tylka, 2011; Simon et al., 2008], wurde hingegen bislang kaum erforscht [Procopio et al., 2006]. Doch auch im fortgeschrittenen Alter können sich Essstörungen gravierend auswirken [Cumella und Kally, 2008a, b].

Psychische Faktoren wie die Überbewertung von Figur und Gewicht beeinflussen das Essverhalten, bilden den Kern der meisten Essstörungen und haben sich als unabhängig von Body Mass Index (BMI) und Alter erwiesen [Hrabosky und Grilo, 2007; Hrabosky et al., 2007; Fairburn und Harrison, 2003]. Bei jungen Frauen kann ein gestörtes Körperbild durch folgende Faktoren verstärkt werden: negative Affekte wie übermäßiges Stressempfinden, Ängstlichkeit, Schamgefühle, Hilflosigkeitsgefühle, Gefühle der Unzulänglichkeit und Depressivität [Stice et al., 2010; Ricciardelli und McCabe, 2004; Taylor und Cooper, 1992; McCarthy, 1990; Watson et al., 1988]. Gemäß Bromberger und Lanza di Scalea [2009] ist zu vermuten, dass eine negative Affektlage das Essverhalten von Frauen im mittleren Lebensalter ebenso beeinflusst wie das von jungen Frauen. Erste Studien zeigen einen Zusammenhang zwischen negativen Affekten und höherer Körperunzufriedenheit bei Frauen im mittleren Lebensalter [Dunkley et al., 2010; Hrabosky und Grilo, 2007; Midlarsky und Nitzburg, 2008]. Dieser Zusammenhang findet sich auch bei Frauen dieser Altersgruppe mit einer diagnostizierten Essstörung [Midlarsky und Nitzburg, 2008; Cumella und Kally, 2008a,b; Forman und Davis, 2005].

Ein Merkmal negativer Affektivität ist eine erhöhte Ängstlichkeit. Dieser Faktor scheint neben Depressivität ein weiterer Prädiktor gestörten Essverhaltens sowohl bei jungen als auch bei älteren Frauen zu sein [Marcus et al., 2007; Procopio et al., 2006]. Als ein weiterer Aspekt negativer Affektivität ist die Zwanghaftigkeit anzuführen. Wie zahlreiche Studien bei jungen Frauen zeigen, besteht ein Zusammenhang zwischen Zwangsstörungen und Essstörungen [Hsu et al., 1993; Polivy und Herman, 2002; Sansone und Sansone, 2010]. Weniger gesichert ist, ob dieser Zusammenhang zwischen Zwanghaftigkeit und pathologischem Essverhalten auch bei Frauen im mittleren Lebensalter besteht. Rogers und Petrie [2001] zeigten einen Zusammenhang zwischen zwanghaftem Verhalten und bulimischen Symptomen bei Frauen im mittleren Lebensalter. Sowohl zwanghaftes Verhalten als auch negative Affektivität sind Konstrukte, bei denen Besorgniskognitionen prädominant sind [Gotlib und Joormann, 2010]. Da eine Überbewertung von Figur und Gewicht mit gestörtem Essverhalten zusammenhängt [Legenbauer et al., 2007], ist zu vermuten, dass diese Kognitionen mit negativer Affek- tivität im mittleren Lebensalter in Zusammenhang stehen könnten.

Sozioökonomische Variablen werden im Zusammenhang mit psychischen Störungen immer wieder diskutiert. Inwiefern der Zusammenhang zwischen Essverhalten und negativer Affektivität durch ein höheres oder niedrigeres Bildungsniveau beeinflusst werden kann, wurde bislang nicht untersucht. Einer Untersuchung von Ahrén-Moonga et al. [2009] zufolge besteht bei jungen Frauen mit einem höheren Bildungsniveau ein höheres Risiko, gestörtes Essverhalten zu entwickeln, als bei jungen Frauen mit einem geringeren Bildungsniveau. Dieser Befund konnte jedoch von Maxwell et al. [2011] nicht bestätigt werden, da sich in dieser Studie bei Frauen mit einem gestörten Essverhalten ein tendenziell niedrigeres Bildungsniveau fand als bei Frauen mit normalem Essverhalten.

Mehrere Studien konnten zeigen, dass die Prävalenzraten für depressive Störungen, die nicht selten in Komorbidität mit Essstörungen auftreten, mit einem niedrigeren sozioökonomischen Status einhergehen [Everson et al., 2002]. Bjelland et al. [2008] konnten zeigen, dass ein höheres Bildungsniveau protektiv für die Entwicklung von Depressionen über die Lebensspanne sein kann.

Zusammenfassend lässt sich feststellen, dass der Einfluss von negativer Affektivität auf figur- und gewichtsbezogene Sorgen bei Frauen im mittleren Lebensalter kaum erforscht ist. Die Befundlage bezüglich des Zusammenhangs zwischen Bildungsniveau und Essstörungen ist nicht eindeutig. Negative Affektivität und insbesondere Depressivität scheint mit einem niedrigeren Bildungsniveau einherzugehen. Unklar ist ebenfalls, ob und gegebenenfalls wie die 3 Konstrukte figurund gewichtsbezogene Sorgen, negative Affektivität und Bildungsniveau bei Frauen im mittleren Lebensalter zusammenhängen. Deshalb haben wir in einer Online-Erhebung das Ausmaß figur- und gewichtsbezogener Sorgen, negativer Affektivität und sozioökonomischer Merkmale an einer großen Stichprobe von Frauen ab 40 Jahren erfasst. Anhand dieses Datensatzes wurden folgende Hypothesen geprüft: 1 . Negative Affektivität, insbesondere Depressivität, Ängstlichkeit und Zwanghaftigkeit hängen positiv mit den figurund gewichtsbezogenen Sorgen zusammen. 2. Der Zusammenhang zwischen negativer Affektivität, insbesondere Depressivität, Ängstlichkeit und Zwanghaftigkeit und den figur- und gewichtsbezogenen Sorgen ist bei Frauen mit einem niedrigeren Bildungsniveau stärker. 3. Der stärkste Prädiktor negativer Affektivität für figur- und gewichtsbezogene Sorgen ist die Depressivität bei Frauen mit einem niedrigeren Bildungsniveau.

\section{Methoden}

\section{Stichprobe}

Die Probandinnen der Online-Erhebung wurden mittels Rundmails sowie verschiedener Inserate auf mehreren Websites von Frauenorganisationen (unter anderem www.frauenlandsgemeinde.ch) kontaktiert. Jede 
Tab. 1. Stichprobenbeschreibung

\begin{tabular}{|c|c|c|c|}
\hline & $\begin{array}{l}\text { Sekundärbildung } \\
(\mathrm{n}=236)\end{array}$ & $\begin{array}{l}\text { Tertiärbildung } \\
(\mathrm{n}=314)\end{array}$ & $\begin{array}{l}\text { Gruppenunterschiede } \\
\text { t-test/Chi }\end{array}$ \\
\hline Alter, Jahre & $M=50,86 ; S D=6,17$ & $M=49,40 ; S D=5,93$ & $\mathrm{~T}=2,81 ; \mathrm{p}=0,005$ \\
\hline BMI, $\mathrm{kg} / \mathrm{m}^{2}$ & $\mathrm{M}=24,23 ; \mathrm{SD}=4,67$ & $\mathrm{M}=22,96 ; \mathrm{SD}=3,65$ & $\mathrm{~T}=3,56 ; \mathrm{p}=0,001$ \\
\hline \multicolumn{4}{|l|}{ Beziehungsstatus } \\
\hline Verheiratet/in einer Beziehung & $\mathrm{N}=196(83,1 \%)$ & $\mathrm{N}=239(76,1 \%)$ & \\
\hline Ledig/getrennt/geschieden & $\mathrm{N}=40(16,9 \%)$ & $\mathrm{N}=75(23,9 \%)$ & $\chi^{2}=6,92 ; p=0,074$ \\
\hline \multicolumn{4}{|l|}{ Anstellungsgrad } \\
\hline $76-100 \%$ & $\mathrm{~N}=77(32,6 \%)$ & $\mathrm{N}=164(52,2 \%)$ & \\
\hline Nicht angestellt/bis zu $75 \%$ & $\mathrm{~N}=159(67,4 \%)$ & $\mathrm{N}=150(47,8 \%)$ & $\chi^{2}=38,86 ; p=<0,001$ \\
\hline EDE-Q_Figur & $\mathrm{M}=1,86 ; \mathrm{SD}=1,62$ & $\mathrm{M}=1,51 ; \mathrm{SD}=1,31$ & $\mathrm{~T}=2,77 ; \mathrm{p}=0,006$ \\
\hline EDE-Q_Gewicht & $\mathrm{M}=1,44 ; \mathrm{SD}=1,44$ & $\mathrm{M}=1,18 ; \mathrm{SD}=1,55$ & $\mathrm{~T}=2,34 ; \mathrm{p}=0,020$ \\
\hline BSI_Depressivität & $\mathrm{M}=1,91 ; \mathrm{SD}=2,93$ & $\mathrm{M}=1,69 ; \mathrm{SD}=2,34$ & $\mathrm{~T}=1,22 ; \mathrm{p}=0,222$ \\
\hline BSI_Ängstlichkeit & $\mathrm{M}=1,91 ; \mathrm{SD}=2,43$ & $\mathrm{M}=1,68 ; \mathrm{SD}=2,01$ & $\mathrm{~T}=1,14 ; \mathrm{p}=0,257$ \\
\hline BSI_Zwanghaftigkeit & $M=3,14 ; S D=2,99$ & $M=2,91 ; S D=2,46$ & $\mathrm{~T}=0,98 ; \mathrm{p}=0,324$ \\
\hline
\end{tabular}

$\mathrm{M}=$ Mittelwert; $\mathrm{SD}=$ Standardabweichung; EDE-Q_Figur = Subskala «figurbezogene Sorgen» des EDE-Q; EDE-Q_Gewicht $=$ Subskala «gewichtsbezogene Sorgen» des EDE-Q; BSI_Depressivitat = Subskala «Depressivität» des BSI; BSI_Ängstlichkeit = Subskala «Ängstlichkeit» des BSI; BSI_Zwanghaftigkeit = Subskala «Zwanghaftigkeit» des BSI; Sekundärbildung = Berufsausbildungen als höchster Bildungsabschluss; Tertiärbildung $=$ Hochschulen als höchster Bildungsabschluss . interessierte Frau ab 40 Jahren konnte an der Untersuchung teilnehmen. Die Stichprobeneigenschaften der Teilnehmerinnen, deren Daten analysiert wurden, sind in Tabelle 1 dargestellt.

Insgesamt haben 1101 Frauen die Internet-Seite der Online-Erhebung aufgerufen. Davon haben 445 Probandinnen den Fragebogen nicht abschließend bearbeitet. 113 Teilnehmerinnen wurden aus folgenden Gründen bei der Datenanalyse nicht berücksichtigt: bestehende Schwangerschaft $(n=4)$, jünger als $40(n=7)$ und älter als 65 Jahre $(n=$ 14), Unvollständigkeit einzelner Fragenantworten $(n=35)$, nach eigenen Angaben aktuelle affektive Störungen, Angststörungen, Essstörungen oder Persönlichkeitsstörungen $(\mathrm{n}=12)$, Antwortmöglichkeit zur Frage nach dem Bildungsstatus mit «andere» angegeben $(\mathrm{n}=16)$. Die Datenanalyse erfolgte an der verbleibenden Stichprobe von $n=550$. Die Gesamtstichprobe wurde in 2 Teilstichproben geteilt. 236 Teilnehmerinnen gaben an, entweder die Hauptschule, Berufsschule, Berufsmatura oder Matura (Abitur) absolviert zu haben und wurden somit der Gruppe «Sekundärbildung» zugeteilt. 314 Probandinnen wählten innerhalb der Antwortmöglichkeiten Fachhochschule, Studium oder Doktorat und wurden somit in die Gruppe «Tertiärbildung» eingeordnet. Diese Einteilung entspricht dem Schweizer Bildungssystem, das sich in die Bildungsabschnitte Vorschule, Primarstufe, Sekundarstufe I, Sekundarstufe II, Tertiärstufe, Quartiärstufe sowie Heil- und Sonderpädagogik gliedert. Die Primarschule und Sekundarstufe I bilden zusammen die obligatorische Schulzeit. Die Sekundarstufe II beinhaltet berufsbildende Ausbildungsgänge (z.B. Berufslehre) sowie allgemeinbildende Ausbildungsgänge (z.B. Gymnasium). Die Tertiärstufe umfasst somit Ausbildungen der höheren Berufsbildung und der Hochschulen.

\section{Vorgehen}

Die Probandinnen erhielten per E-Mail den Link zur Internet-Seite der Online-Erhebung auf «Unipark» (www.unipark.info/1-0-home.htm). Auf der Internet-Seite wurden die potenziellen Teilnehmerinnen zuerst über die Studie informiert. Anschließend erklärten sie ihr Einverständnis zu den Teilnahmebedingungen. Danach wurden zunächst soziodemographische Angaben wie Alter, BMI, Beziehungsstatus, Anstellungsgrad und Bildungsstatus erhoben, bevor in randomisierter Reihenfolge die Fragebögen zum Essverhalten («Eating Disorder Questionnaire», EDE-Q, siehe unten) und allgemeinen psychischen Merkmalen («Brief Symptom Inventory», BSI, siehe unten) folgten. Die Gesamtbearbeitung der Fragen dauerte ungefähr $15 \mathrm{~min}$. Die Studie wurde in Übereinstimmung mit der «Deklaration von Helsinki» durchgeführt.

\section{Negative Affektivität}

Die negative Affektivität wurde mit der deutschen Version des BSI erfasst [Derogatis, 1993; Franke, 2000]. Der BSI beinhaltet 53 Items und ist ein Instrument zur Erfassung der Beeinträchtigung des subjektiven Wohlbefindens aufgrund körperlicher und psychischer Symptome. Das Inventar besteht aus 9 Subskalen: Somatisierung, Zwanghaftigkeit, Unsicherheit im Sozialkontakt, Depressivität, Ängstlichkeit, Aggressivität, phobische Angst, paranoides Denken und Psychotizismus. Zusätzlich ermöglichen 3 Globalwerte Informationen über das Antwortverhalten bei allen Fragen des BSI. Jede Frage dieses Instruments kann auf einer 5-stufigen Likert-Skala beantwortet werden. Für die Beantwortung der Fragestellungen dieser Studie wurden nur die Subskalen Depressivität, Ängstlichkeit und Zwanghaftigkeit verwendet. Das Cronbachs alpha dieser Skalen war akzeptabel (BSI_Depressivität $=0,827$; BSI_Ängstlichkeit $=0,712$; BSI $_{\text {Zwanghaftigkeit }}$ $=0,742$ ).

\section{Figur- und gewichtsbezogene Sorgen}

Die figur- und gewichtsbezogenen Sorgen wurde mittels der deutschen Version des EDE-Q erhoben [Fairburn und Beglin, 1994; Hilbert et al., 2007]. Der EDE-Q ist ein Erhebungsinstrument mit 36 Items, und die gestellten Fragen beziehen sich auf das Essverhalten der letzten 28 Tage. Der Fragebogen beinhaltet 4 Subskalen, welche das Ausmaß gezügelten Essverhaltens sowie ess-, gewichts- und figurbezogener Sorgen abbilden. Die Subskalen «gezügeltes Essverhalten» und «essensbezogene Sorgen» dienen der Erfassung verhaltensbezogener Aspekte gestörten Essverhaltens. Zusätzlich lässt sich ein Gesamtwert aus allen Subskalen bilden. Alle Items werden auf einer 7-stufigen Likert-Skala beantwortet, wobei höhere Werte eine stärkere essensbezogene Problematik repräsentieren. Zusätzlich werden die Häufigkeiten von gewichtsreduzierenden Maßnahmen wie z.B. die Häufigkeit von «Erbrechen» erfasst. Cronbachs alpha der untersuchten Subskalen war akzeptabel $\left(\mathrm{EDE}^{-Q_{\text {Gewicht }}}=0,77\right.$; EDE- $Q_{\text {Figur }}=0,89$ ). 


\begin{tabular}{lllllllll}
\hline & 1 & 2 & 3 & 4 & 5 & M & SD & Spanne \\
\hline $\begin{array}{l}\text { Sekundärbildung } \\
\text { 1 EDE-Q_Figur }\end{array}$ & & & & & & & & \\
2 EDE-Q_Gewicht & 0,92 & & & & & 1,85 & 1,61 & $0-6$ \\
3 BSI_Depressivität & 0,48 & 0,48 & & & & 1,44 & 1,43 & $0-6$ \\
4 BSI_Ängstlichkeit & 0,34 & 0,34 & 0,55 & & & 1,91 & 2,92 & $0-18$ \\
5 BSI_Zwanghaftigkeit & 0,44 & 0,41 & 0,61 & 0,61 & & 3,14 & 2,42 & $0-18$ \\
Tertiärbildung & & & & & & & & $0-18$ \\
1 EDE-Q_Figur & & & & & & 1,51 & 1,31 & $0-5,25$ \\
2 EDE-Q_Gewicht & 0,90 & & & & & 1,18 & 1,15 & $0-5,50$ \\
3 BSI_Depressivität & 0,32 & 0,25 & & & & 1,63 & 2,35 & $0-14$ \\
4 BSI_Ängstlichkeit & 0,28 & 0,19 & 0,54 & & & 1,68 & 2,01 & $0-13$ \\
5 BSI_Zwanghaftigkeit & 0,35 & 0,28 & 0,54 & 0,59 & & 2,91 & 2,46 & $0-13$ \\
\hline
\end{tabular}

Sofern nichts anderes vermerkt, waren alle Koeffizienten auf dem $p=0,001$-Niveau signifikant. $\mathrm{M}=$ Mittelwert; $\mathrm{SD}$ = Standardabweichung; EDE-Q_Figur = Subskala «figurbezogene Sorgen» des EDE-Q; EDE-Q_Gewicht = Subskala «gewichtsbezogene Sorgen» des EDE-Q; BSI_Depressivität = Subskala «Depressivität» des BSI; BSI_Ängstlichkeit = Subskala «Ängstlichkeit» des BSI; BSI_Zwanghaftigkeit = Subskala «Zwanghaftigkeit» des BSI; Sekundärbildung = Berufsausbildungen als höchster Bildungsabschluss; Tertiärbildung = Hochschulen als höchster Bildungsabschluss.

\section{Datenanalyse}

Zur Prüfung von Zusammenhängen zwischen dem negativen Affekt und den figur- und gewichtsbezogenen Sorgen wurden bivariate Korrelationen in Abhängigkeit vom Bildungsniveau errechnet. Zudem wurde geprüft, ob sich die Stärke der Korrelationen signifikant voneinander unterscheidet. Abschließend wurde für die beiden Bildungsgruppen und die Variablen figur- und gewichtsbezogene Sorgen eine schrittweise multiple Regressionsanalyse mit dem Ziel durchgeführt, den besten Prädiktor für die jeweilige abhängige Variable zu bestimmen.

\section{Ergebnisse}

\section{Der Zusammenhang zwischen negativer Affektivität und} figur- und gewichtsbezogenen Sorgen

Die Korrelationen zwischen Depressivität, Ängstlichkeit und Zwanghaftigkeit und den figur- und gewichtsbezogenen Sorgen waren alle positiv signifikant. Dieses Resultat zeigte sich ebenfalls für die Korrelationen, welche separat für die Gruppe mit Sekundärbildung bzw. Tertiärbildung ermittelt wurden. In Tabelle 2 ist die Korrelationsmatrix aller verwendeten Variablen dargestellt. Die Korrelationsunterschiede zwischen Depressivität und den figurbezogenen Sorgen sind signifikant zwischen der Gruppe mit der Sekundärbildung und der Gruppe mit der Tertiärbildung $(\mathrm{Z}=2,21, \mathrm{p}=0,027)$. Ebenso sind die Korrelationen zwischen Depressivität und den gewichtsbezogenen Sorgen in beiden signifikant unterschiedlich $(Z=3,09 ; p=0,002)$. Die Korrelationen zwischen Ängstlichkeit und den figurbezogenen Sorgen zeigen keine signifikanten Unterschiede $(Z=0,076 ; p=0,0443)$. Ebenfalls unterscheiden sich die Korrelationen zwischen Ängstlichkeit und den gewichtsbezogenen Sorgen nicht in den beiden Bildungsgruppen $(Z=1,86 ; p=0,062)$. Schließlich konnte kein signifikanter
Unterschied zwischen den Korrelationen Zwanghaftigkeit und figurbezogene Sorgen $(Z=1,232 ; p=0,218)$ und Zwanghaftigkeit und gewichtsbezogene Sorgen $(\mathrm{Z}=1,71, \mathrm{p}=0,088)$ zwischen den beiden Bildungsniveaus nachgewiesen werden.

\section{Prädiktoren figur- und gewichtsbezogener Sorgen}

Die unabhängigen Variablen Depressivität, Ängstlichkeit und Zwanghaftigkeit wurden als Prädiktoren in einem schrittweisen multiplen Regressionsmodell für jede Bildungsgruppe getestet. Für die Gruppe der Frauen mit einem Sekundärbildungsabschluss fanden sich folgende Resultate: Die schrittweise multiple Regressionsanalyse mit den unabhängigen Variablen Depressivität, Ängstlichkeit und Zwanghaftigkeit und der abhängigen Variable gewichtsbezogene Sorgen zeigte, dass die 3 Variablen insgesamt 25,5\% der Varianz aufklärten, wobei Depressivität der beste Prädiktor war. Die schrittweise multiple Regressionsanalyse mit der abhängigen Variable figurbezogene Sorgen zeigte, dass die genannten unabhängigen Variablen 25,6\% der Varianz aufklärten, wobei sich Depressivität und Zwanghaftigkeit als beste Prädiktoren erwiesen haben.

Die Auswertung für die Gruppe mit einem Tertiärbildungsabschluss ergab folgende Resultate: Die unabhängigen Variablen klärten insgesamt 9,4\% der abhängigen Variable gewichtsbezogene Sorgen auf, wobei Depressivität und Zwanghaftigkeit die besten Prädiktoren waren. Die Varianzaufklärung für die figurbezogenen Sorgen betrug insgesamt 15,0\%, wobei Depressivität und Zwanghaftigkeit ebenfalls die besten Prädiktoren waren. Die Ergebnisse der multiplen Regressionsanalysen sind in Tabelle 3 dargestellt. 
Tab. 3. Regressionsanalytische Befunde zum Einfluss von negativer Affektivität auf figurund gewichtsbezogene Sorgen

\begin{tabular}{|c|c|c|c|c|c|}
\hline & $\mathrm{R}$ & $\mathrm{R} 2$ & $\mathrm{~B}$ & $\beta$ & $\mathrm{p}$ \\
\hline \multicolumn{6}{|l|}{ EDE-Q_Figur } \\
\hline BSI_Depressivität & 0,233 & 0,230 & 0,187 & 0,339 & $0,000^{c}$ \\
\hline BSI_Ängstlichkeit & 0,241 & 0,235 & 0,014 & 0,021 & 0,779 \\
\hline BSI_Zwanghaftigkeit & 0,266 & 0,256 & 0,117 & 0,217 & $0,006^{\mathrm{b}}$ \\
\hline \multicolumn{6}{|l|}{ Tertiärbildung } \\
\hline BSI_Depressivität & 0,318 & 0,101 & 0,089 & 0,160 & $0,016^{\mathrm{a}}$ \\
\hline BSI_Ängstlichkeit & 0,342 & 0,117 & 0,032 & 0,049 & 0,474 \\
\hline BSI_Zwanghaftigkeit & 0,387 & 0,150 & 0,126 & 0,239 & $0,001^{\mathrm{b}}$ \\
\hline \multicolumn{6}{|l|}{$\begin{array}{l}\text { EDE-Q_Gewicht } \\
\text { Sekundärbildung }\end{array}$} \\
\hline BSI_Depressivität & 0,486 & 0,236 & 0,181 & 0,369 & $0^{\mathrm{c}}$ \\
\hline BSI_Ängstlichkeit & 0,494 & 0,244 & 0,029 & 0,048 & 0,519 \\
\hline BSI_Zwanghaftigkeit & 0,505 & 0,255 & 0,071 & 0,148 & 0,061 \\
\hline \multicolumn{6}{|l|}{ Tertiärbildung } \\
\hline BSI_Depressivität & 0,252 & 0,064 & 0,071 & 0,144 & $0,036^{\mathrm{a}}$ \\
\hline BSI_Ängstlichkeit & 0,260 & 0,068 & $-0,009$ & $-0,016$ & 0,821 \\
\hline BSI_Zwanghaftigkeit & 0,307 & 0,094 & 0,100 & 0,214 & $0,003^{\mathrm{b}}$ \\
\hline \multicolumn{6}{|c|}{$\begin{array}{l}\mathrm{R}=\text { Korrelationskoeffizient; } \mathrm{R} 2=\text { Determinationskoeffizient; } \mathrm{B}=\text { Regressionskoeffizient; } \\
\beta=\text { standardisierter Regressionskoeffizient } \\
\text { EDE-Q_Figur = Subskala «figurbezogene Sorgen» des EDE-Q; Sekundärbildung = Berufsaus- } \\
\text { bildungen als höchster Bildungsabschluss; BSI_Depressivitat = Subskala «Depressivität» des BSI; } \\
\text { BSI_Ängstlichkeit = Subskala «Ängstlichkeit» des BSI; BSI_Zwanghaftigkeit = Subskala «Zwanghaftigkeit» } \\
\text { des BSI; Tertiärbildung = Hochschulen als höchster Bildungsabschluss; EDE-Q_Gewicht = Subskala } \\
\text { «gewichtsbezogene Sorgen» des EDE-Q } \\
\text { ap }<0,05 ; \text { bp }<0,01 ; \text { cp }<0,001 \text {. }\end{array}$} \\
\hline
\end{tabular}

Für alle linearen Regressionsmodelle wurden Angaben zur Kollinearitätsdiagnose herangezogen. Die Kollinearitätsstatistik der linearen Regressionsanalyse mit den Prädiktoren Depressivität, Zwanghaftigkeit und Ängstlichkeit und der abhängigen Variable figurbezogene Sorgen ergaben VIF-Werte von 2,031-2,253. Die Kollinearitätsstatistik der linearen Regressionsanalyse mit den Prädiktoren Depressivität, Zwanghaftigkeit und Ängstlichkeit und der Variable abhängige gewichtsbezogenen Sorgen zeigen dieselben VIF-Werte. Da die VIF-Werte die kritische Grenze von 10 nicht überschreiten, kann davon ausgegangen werden, dass keine Multikollinearität vorliegt.

\section{Diskussion}

Gemäß der Resultate unserer Erhebung hängen Depressivität, Ängstlichkeit und Zwanghaftigkeit bei Frauen im mittleren Lebensalter signifikant mit figur- und gewichtsbezogenen Sorgen zusammen. Der Zusammenhang zwischen Depressivität und figur- und gewichtsbezogenen Sorgen ist jedoch bei Frauen mit niedrigerem Bildungsniveau stärker als bei Frauen mit höherem Bildungsniveau. Weiterhin zeigte sich, dass von den untersuchten Dimensionen negativer Affektivität das Ausmaß an Depressivität und Zwanghaftigkeit die stärksten
Prädiktoren für figur- und gewichtsbezogene Sorgen sind, unabhängig vom Bildungsniveau.

Die Ergebnisse weisen darauf hin, dass nicht nur bei jungen Frauen negative Affekte auffälliges Essverhalten beeinflussen [Stice et al., 2010; McCarthy, 1990; Taylor und Cooper, 1992], sondern, dass sich dieser Zusammenhang auch bei Frauen im mittleren Lebensalter zeigt. Aufgrund der vorliegenden Querschnittsuntersuchung lässt sich die Kausalität zwischen negativen Affekten, Körperunzufriedenheit und auffälligem Essverhalten allerdings nicht klären.

In dieser Studie konnte ferner gezeigt werden, dass der Zusammenhang zwischen Depressivität und figur- und gewichtsbezogenen Sorgen bei Frauen mit einem niedrigeren Bildungsniveau stärker ausgeprägt ist als bei Frauen mit einem höheren Bildungsniveau. Dieser Befund steht im Einklang mit der einschlägigen Literatur, welche einen Zusammenhang zwischen Depressivität und geringerer Bildung feststellt [Chazelle et al., 2011].

Depressivität und Zwanghaftigkeit scheinen bei Frauen im mittleren Lebensalter deutliche Prädiktoren figur- und gewichtsbezogener Sorgen zu sein. Mehrfach konnten Studien zeigen, dass sich bei Frauen eine hohe Komorbidität zwischen affektiven Störungen und Essstörungen findet [Lewinsohn et al., 2000; Santos et al., 2007; Zaider et al., 2000]. Zusätzlich zeigte sich in einer neueren Studie, dass depressive Störungen 
und Bulimia nervosa in einem reziproken Zusammenhang stehen [Presnell et al., 2009]. Unser Ergebnis lässt sich in die bisherige Literatur einordnen. Die Ergebnisse können dahingehend interpretiert werden, dass bei Therapie von auffälligem bis hin zu gestörtem Essverhalten die psychischen Beschwerden nicht außer Acht gelassen, sondern mitbehandelt werden sollten [Bushnell et al., 1994; Sloan et al., 2005; Burton et al., 2007].

Eine ähnliche Interpretation liegt auch für den Zusammenhang zwischen Zwanghaftigkeit und auffälligem Essverhalten nahe. Zwanghafte Störungen und gestörtes Essverhalten weisen eine ähnliche Pathogenese auf bzw. eine Störung kann einen Risikofaktor für die Entwicklung der anderen darstellen [Altman und Shankman, 2009]. In diesem Zusammenhang wird diskutiert, dass Perfektionismus eine beiden Störungsgruppen gemeinsame Persönlichkeitseigenschaft darstellt, welche den Zusammenhang zwischen Zwangsstörungen und Essstörungen mediieren könnte [Wade et al., 2008]. Da zwanghaftes Verhalten das Kontrollbedürfnis befriedigt [Altman und Shankman, 2009] und gestörtes Essverhalten unter anderem den Kontrollverlust über das Essverhalten thematisiert [Legenbauer et al., 2007], liegt es nahe, dass diese beiden Konstrukte eng verknüpft sind.

Ängstlichkeit scheint nicht einer der Hauptprädiktoren für figur- und gewichtsbezogene Sorgen zu sein. Ein Grund dafür könnte sein, dass depressive Erkrankungen und Zwangserkrankungen häufiger als Komorbiditäten bei Essstörungen auftreten als Angststörungen [APA, 2000].

Bei der Interpretation dieser Resultate sollten die Limitationen der vorliegenden Studie berücksichtigt werden. Eine Online-Erhebung hat verschiedene Vorteile, wie die Möglichkeit, eine große Anzahl von Frauen zu erreichen, wobei nicht alle interessierten Frauen den Fragebogen bis zum Ende be- arbeiten. Im Fall unserer Studie lag die Abbruchquote bei $45 \%$. Unsere Abbruchrate entspricht in etwa derjenigen, die Musch und Reips [2000] sowie Reips [2002] für Online-Studien berichtet haben (ca. 35\%). Allerdings sind folgende Nachteile bei diesem Vorgehen zu bedenken: Die Generalisierung unserer Resultate auf die Gesamtpopulation von Frauen im mittleren Lebensalter ist limitiert, weil das Interesse unserer Stichprobe vielleicht besonders groß sein könnte für das Wissen rund um den Körper, das Essverhalten und sogar Essstörungen. Zudem wird der Umgang mit dem Internet vorausgesetzt.

Abschließend lässt sich festhalten, dass an unserer Studie Frauen teilnahmen, die nach eigenen Angaben psychisch gesund waren. Die Mittelwerte in den Skalen figur- und gewichtsbezogene Sorgen entsprechen einer gesunden, nicht essgestörten Population [Hilbert und Tuschen-Caffier, 2006]. Somit können keine generellen Schlussfolgerungen über gestörtes Essverhalten oder Essstörungen in dieser Altersgruppe gezogen werden. Vielmehr zeigt unsere Studie einerseits, dass figur- und gewichtsbezogene Sorgen bei gesunden Frauen im mittleren Lebensalter mit negativer Affektivität assoziiert sind und andererseits, dass dieser Zusammenhang vom Bildungsniveau beeinflusst wird. Durch diese und folgende Studien könnten somit spezifische Risikogruppen für gestörtes Essverhalten eruiert werden. Das Bewusstsein für Risikogruppen könnte bei der Frühindikation bzw. Prävention von Essstörungen sinnvoll eingesetzt werden.

\section{Disclosure Statement}

Es bestehen keine finanziellen oder beruflichen Verpflichtungen, aus denen sich für die Autoren ein Interessenskonflikt ergeben könnte.

\section{Literatur}

Ahrén-Moonga J, Silverwood R, Klinteberg B, Koupil I: Association of higher parental and grandparental education and higher school grades with risk of hospitalization of Eating Disorders in females. Am J Epidemiol 2009;170:566-575.

Augustus-Horvath CL, Tylka TL: The acceptance model of intuitive eating: a comparison of women in emerging adulthood, early adulthood, and middle adulthood. J Couns Psychol 2011;58:110-125.

Altman SE, Shankman SA: What is the association between obsessive-compulsive disorder and eating disorders? Clin Psychol Rev 2009;29:638-646.

American Psychiatric Association (APA): Diagnostic and Statistical Manual of Mental Disorders. Textrevision, ed. 4). Washington DC, American Psychiatric Association, 2000.

Bjelland I, Krokstad S, Mykletun A, Dahl AA, Tell GS, Tambs K: Does a higher educational level protect against anxiety and depression? The HUNT study. Soc Sci Med 2008;66:1334-1345.

Bromberger JT, Lanza di Scalea TL: Longitudinal associations between depression and functioning in midlife women. Maturitas 2009;64:145-159.
Bushnell JA, Wells JE, McKenzie JM, Hornblow AR, Oakley-Browne MA, Joyce PR: Bulimia comorbidity in the general population and in the clinic. Psychol Med 1994;24:605-611.

Burton EM, Stice E, Bearman SK, Rohde P: An experimental test of the affect-regulation model of bulimic symptoms and substance use: an affective intervention. Int J Eat Disord 2007;40:27-36.

Chazelle E, Lemogne C, Morgan K, Kelleher CC, Chastang JF and Niedhammer I: Explanations of educational differences in major depression and generalised anxiety disorder in the Irish population. J Affect Disord 2011;134:304-314.

Cumella EJ, Kally Z: Profile of 50 women with midlife-onset eating disorders. Eat Disord 2008a; 16:193-203.

Cumella EJ, Kally Z: Comparison of middle-age and young women inpatiens with eating disorders. Eat Weight Disord 2008b;13:183-190.

Derogatis, LR: BSI Brief Symptom Inventory. Administration, Scoring, and Procedures Manual, ed 4. Minneapolis, MN, National Computer Systems, 1993.
Dunkley DM, Masheb RM, Grilo CM: Childhood maltreatment, depressive symptoms, and body dissatisfaction in patients with binge eating disorder: the mediating role of self-criticsm. Int J Eat Disord 2010;43:274-281.

Everson SA, Maty SC, Lynch JW, Kaplan GA: Epidemiologic evidence for the relation between socioeconomic status and depression, obesity, and diabetes. J Psychosom Res 2002;53:891-895.

Fairburn CG, Beglin SJ: Assessment of eating disorders: interview or self-report questionnaire? Int J Eat Disord 1994;16:363-370.

Fairburn CG, Harrison PJ: Eating disorders. Lancet 2003;361:407-416.

Forman M, Davis WN: Characteristics of middle-aged women in inpatient treatment for eating disorders. Eating Disord 2005;13:231-243.

Franke GH: BSI - Brief Symptom Inventory von L.R. Derogatis. Göttingen, Hogrefe, 2000.

Gotlib IH, Joormann J: Cognition and depression: current status and future directions. Annu Rev Clin Psychol 2010;6:285-312. 
Hilbert A, Tuschen-Caffier B: Eating Disorder Examination - Questionnaire: Deutschsprachige Übersetzung. Münster, Verlag für Psychotherapie, 2006.

Hilbert A, Tuschen-Caffier B, Karwautz A, Niederhofer H, Munsch S: Eating Disorder ExaminationQuestionnaire. Evaluation der deutschsprachigen Übersetzung. Diagnostica 2007;53:144-154.

Hrabosky JI, Grilo CM: Body image and eating disordered behaviour in a community sample of Black and Hispanic women. Eat Behav 2007;8:106-114.

Hrabosky JI, Masheb, RM, White MA, Grilo CM Overevaluation of shape and weight in binge eating disorder. J Consult Clin Psychol 2007;75:175-180.

Hsu LK, Kaye W, Weltzin T: Are the eating disorders related to obsessive compulsive disorder? Int J Eat Disord 1993;14:305-318.

Legenbauer T, Vocks S, Schütt-Strömel S: Dysfunktionale Kognitionen bei Essstörungen: Welche Inhaltsbereiche lassen sich unterscheiden? Z Klin Psychol Psychother 2007;36:207-215.

Lewinsohn PM, Striegel-Moore RH, Seeley JR: Epidemiology and natural course of eating disorders in young women from adolescence to young adulthood. JAACP 2000;39:1284-1292.

Marcus MD, Bromberger JT, Wie HL, Brown C, Kravitz HM: Prevalence and selected correlations of eating disorder symptoms among a multiethnic community sample of midlife women. Ann Behav Med 2007;33:269-277.

Maxwell MM, Thornton LM, Root TL, Poyastro Pinheiro A, Strober M, Brandt H, Crawford S, Crow S, Fichter MM, Halmi KA, Johnson C, Kaplan AS, Keel P, Klump KL, LaVia M, Mitchell JE, Plotnicov K, Rotondo A, Woodside DB, Berrettini WH, Kaye WH, Bulik CM: Life beyond the eating disorder: education, relationships, and reproduction. In J Eat Disord 2011;44:225-232.
McCarthy M: The thin ideal, depression and eating disorders in women. Behav Res and Therap 1990; 28:205-215.

Midlarsky E, Nitzburg G: Eating disorders in middleaged women. J Gen Psychol 2008;135:393-407.

Musch J, Reips U-D: A brief history of web experimenting; in Birnbaum MH (ed): Psychological Experiments on the Internet. San Diego, CA, Academic Press, 2000, pp 61-85.

Polivy J, Herman CP: Causes of eating disorders. Annu Rev Psychol 2002;53:187-213.

Presnell K, Stice E, Seidel A, Madeley MC: Depression and eating pathology: prospective reciprocal relations in adolescents. Clin Psychol Psychother 2009;16:357-365.

Procopio CA, Holm-Denoma JM, Gordon KH, Joiner TE: Two-three-year stability and interrelations of bulimotypic indicators and depressive and anxious symptoms in middle-aged women. Int J Eat Disord 2006;39:312-319.

Reips UD: Standards for Internet-based experimenting. Exp Psychol 2002;49:243-256.

Ricciardelli LA, McCabe MP: A biopsychosocial model of disordered eating and the pursuit of muscularity in adolescent boys. Psychol Bull 2004;130: 179-205.

Rogers RL, Petrie TA: Psychological correlates of anorexic and bulimic symptomatology. J Couns Dev 2001;79:178-187.

Sansone RA, Sansone LA: Personality disorders as risk factors for eating disorders: clinical implications. Nutr Clin Pract 2010;25:116-121.
Santos M, Richards CS, Bleckley MK: Comorbidity between depression and disordered eating in adolescents. Eat Behav 2007;8:440-449.

Schmidt U. Aetiology of eating disorders in the 21(st) century: new answers to old questions. Eur Child Adolesc Psychiatry 2003;12(suppl 1):130-137.

Simon GE, Ludman EJ, Linde JA, Operskalski BH, Ichikawa L, Rohde P, Finch EA, Jeffery RW: Association between obesity and depression in middleaged women. Gen Hosp Psychiatry 2008;30:32-39.

Sloan DM, Mizes JS, Epstein EM: Empirical classification of eating disorders 2005;6:53-62.

Stice E, Ng J, Shaw H: Risk factors and prodromal eating pathology. J Child Psychol Psychiatry 2010;51: 518-525.

Taylor MJ, Cooper PJ: An experimental study of the effect of mood on body size perception. Beh Res Ther 1992;30:53-58.

Wade TD, Tiggemann M, Bulik CM, Fairburn CG, Wray NR, Martin NG: Shared temperament risk factors for anorexia nervosa: a twin study. Psychosom Med 2008;70:239-244.

Watson D, Clark LA, Tellegen A: Development and validation of brief measures of positive and negative affect: the PANAS scales. J Pers Soc Psychol 1988;54:1063-1070.

Zaider TI, Johnson JG, Cocknell SJ: Psychiatric comorbidity associated with eating disorder symptomatology among adolescents in the community. Int J Eat Disord 2000;28:405-414. 